\title{
An Autonomous Onboard Targeting Algorithm Using Finite Thrust Maneuvers
}

\author{
Sara K. Scarritt; Belinda G. Marchand ${ }^{\ddagger}$ Michael W. Weeks ${ }^{\ddagger}$
}

\begin{abstract}
I. Abstract
In earlier investigations, the adaptation and implementation of a modified two-level corrections process as the onboard targeting algorithm for the Trans-Earth Injection phase of Orion is presented. The objective of that targeting algorithm is to generate the times of ignition and magnitudes of the required maneuvers such that the desired state at entry interface is achieved. In an actual onboard flight software implementation, these times of ignition and maneuvers are relayed onto Flight Control for command and execution. Although this process works well when the burn durations or burn arcs are small, this might not be the case during a contingency situation when lower thrust engines are employed to perform the maneuvers. Therefore, a new version of the modified two-level corrections process is formulated to handle the case of finite burn arcs. This paper presents the development and formulation of that finite burn modified two-level corrections process which can again be used as an onboard targeting algorithm for the Trans-Earth Injection phase of Orion. Additionally, performance results and a comparison between the two methods are presented. The finite burn two-level corrector formulation presented here ensures the entry constraints at entry interface are still met without violating the available fuel budget, while still accounting for much longer burn times in its design.
\end{abstract}

\section{Introduction}

T JDER nominal operational conditions, the Crew Module's (CM) 33,361 N main engine performs the 3burn Trans-Earth Injection (TEI) sequence. However, the spacecraft must also have the ability to target and execute the maneuvers to return the crew safely to Earth using the backup auxiliary engines. Since the total thrust of these eight (8) auxiliary engines is $4,400 \mathrm{~N}$, the burn durations are naturally much longer. By way of comparison, that yields a burn duration of 5.5 minutes for the baseline TEI-1 maneuver with the main engine, and 55 minutes to perform the same maneuver with the auxiliary engines.

In the next section of this paper, the formulation of the finite burn algorithm is presented. Next, the performance results and a comparison between the two methods is discussed. For all the runs considered, the same initial conditions and same entry targets are used. An optimal trajectory will be generated for each case to facilitate a comparison with the targeting algorithm results.

\section{Finite Burn Targeting Algorithm}

\section{A. Level I Process}

The Level I process is a differential correction scheme that seeks to identify the transfer arc between two position vectors by adjusting the departure velocity. Consider a segment of the trajectory whose endpoints (patch points) are denoted by $k-1$ and $k$, as shown in Figure 1. In a Level I process that employs finite burns rather than impulsive maneuvers, the burn arc is considered to be a subsegment of the arc between patch points $k-1$ and $k$. The end of the burn occurs at point $T$. In identifying finite burn arcs, it is necessary to consider an augmented state vector $\left[\begin{array}{lllll}\bar{R}_{k} & \bar{V}_{k} & m_{k} & \dot{m}_{g_{k}} & \bar{u}_{k}\end{array}\right]^{T}$, where $m_{k}, \dot{m}_{g_{k}}$, and $\bar{u}_{k}$

\footnotetext{
*Graduate Student, Aerospace Engineering and Engineering Mechanics, The University of Texas at Austin, 210 E. 24 th St., Austin, TX 78712.

${ }^{\dagger}$ Assistant Professor, Aerospace Engineering and Engineering Mechanics, The University of Texas at Austin, 210 E. 24th St., Austin, TX 78712.

¥GNC Engineer, Aeroscience and Flight Mechanics Division, NASA JSC, 2101 NASA Pkwy. Houston, Texas 77058 / EG-6.
} 


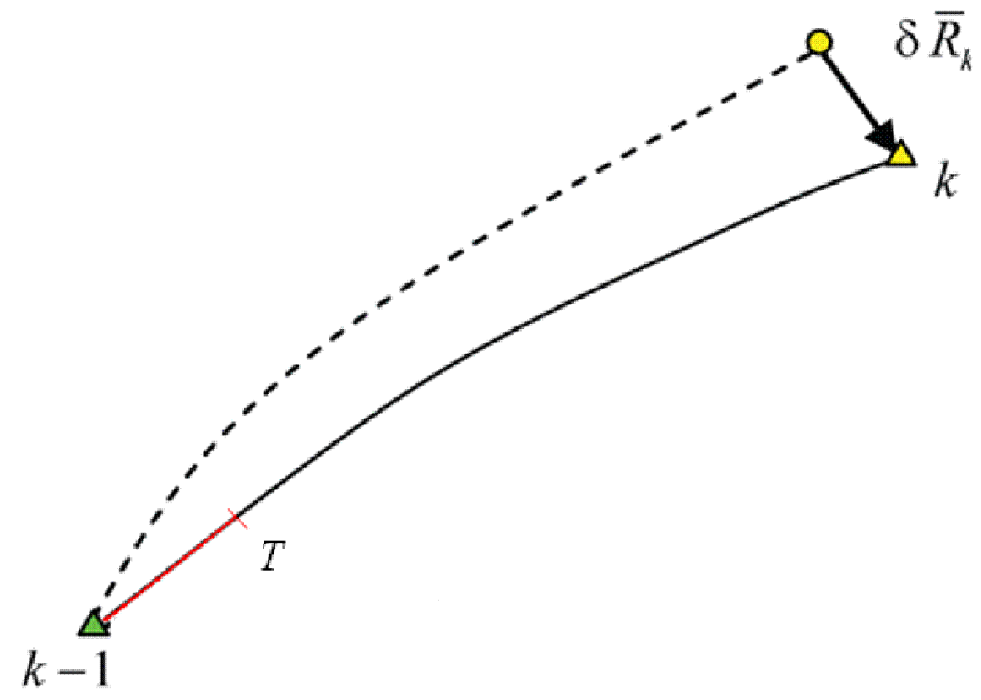

Figure 1. Level 1 Process

represent the spacecraft mass, the propellant flow rate, and the thrust direction associated with patch point $k$, respectively. The goal is to identify a relation between the target, which is the terminal position vector at point $k\left(\bar{R}_{k}\right)$, and the control variables. The control variables are the thrust direction $\left(\bar{u}_{k-1}=\bar{u}_{k}\right)$ and the time at the end of the burn $\left(t_{T}\right)$. As in the impulsive formulation, the state transition matrix is partitioned into sub-matrices corresponding to each state. The variational equations for each subsegment are then

$$
\begin{aligned}
& {\left[\begin{array}{c}
\delta \bar{R}_{T}-\bar{V}_{T}^{-} \delta t_{T} \\
\delta \bar{V}_{T}^{-}-\bar{a}_{T}^{-} \delta t_{T} \\
\delta m_{T}^{-}+\dot{m}_{g_{T}}^{-} \delta t_{T} \\
\delta \dot{m}_{g_{T}}^{-}-\ddot{m}_{g_{T}}^{-} \delta t_{T} \\
\delta \bar{u}_{T}^{-}-\dot{\bar{u}}_{T}^{-} \delta t_{T}
\end{array}\right]=} \\
& {\left[\begin{array}{ccccc}
A_{T, k-1} & B_{T, k-1} & E_{T, k-1} & F_{T, k-1} & G_{T, k-1} \\
C_{T, k-1} & D_{T, k-1} & H_{T, k-1} & I_{T, k-1} & J_{T, k-1} \\
K_{T, k-1} & L_{T, k-1} & M_{T, k-1} & N_{T, k-1} & O_{T, k-1} \\
P_{T, k-1} & Q_{T, k-1} & R_{T, k-1} & S_{T, k-1} & T_{T, k-1} \\
U_{T, k-1} & V_{T, k-1} & W_{T, k-1} & X_{T, k-1} & Y_{T, k-1}
\end{array}\right]\left[\begin{array}{c}
\delta \bar{R}_{k-1}-\bar{V}_{k-1}^{+} \delta t_{k-1} \\
\delta \bar{V}_{k-1}^{+}-\bar{a}_{k-1}^{+} \delta t_{k-1} \\
\delta m_{k-1}^{+}+\dot{m}_{g_{k-1}}^{+} \delta t_{k-1} \\
\delta \dot{m}_{g_{k-1}}^{+}-\ddot{m}_{g_{k-1}}^{+} \delta t_{k-1} \\
\delta \bar{u}_{k-1}^{+}-\dot{\bar{u}}_{k-1}^{+} \delta t_{k-1}
\end{array}\right]}
\end{aligned}
$$

for the burn subsegment, and

$$
\left[\begin{array}{c}
\delta \bar{R}_{k}-\bar{V}_{k}^{-} \delta t_{k} \\
\delta \bar{V}_{k}^{-}-\bar{a}_{k}^{-} \delta t_{k}
\end{array}\right]=\left[\begin{array}{cc}
A_{k, T} & B_{k, T} \\
C_{k, T} & D_{k, T}
\end{array}\right]\left[\begin{array}{c}
\delta \bar{R}_{T}-\bar{V}_{T}^{+} \delta t_{T} \\
\delta \bar{V}_{T}^{+}-\bar{a}_{T}^{+} \delta t_{T}
\end{array}\right]
$$

for the subsequent coasting subsegment.

For this formulation, both the initial and final times of the arc $\left(t_{k-1}\right.$ and $\left.t_{k}\right)$ are fixed, as are the initial position $\bar{R}_{k-1}$, velocity $\bar{V}_{k-1}$, and mass $m_{k-1}$. The mass flow rate, $\dot{m}_{g_{k-1}}$, is also fixed. It is important to note that $\bar{V}_{T}^{+}=\bar{V}_{T}^{-}$(and therefore $\delta \bar{V}_{T}^{+}=\delta \bar{V}_{T}^{-}$), and that $\delta \bar{V}_{T}^{+}-\bar{a}_{T}^{+} \delta t_{T}=\delta \bar{V}_{T}^{-}-\bar{a}_{T}^{-} \delta t_{T}+\left(\bar{a}_{T}^{-}-\bar{a}_{T}^{+}\right) \delta t_{T}$. Incorporating these substitutions, the first two variational equations from Equation (1) and Equation (2) can be combined to give an expression for $\delta \bar{R}_{k}$;

$$
\delta \bar{R}_{k}=A_{k, T} G_{T, k-1} \delta \bar{u}_{k-1}^{+}+B_{k, T}\left(J_{T, k-1} \delta \bar{u}_{k-1}^{+}+\left(\bar{a}_{T}^{-}-\bar{a}_{T}^{+}\right) \delta t_{T}\right) .
$$


Rearranging this equation into matrix form gives

$$
\delta \bar{R}_{k}=\left[\begin{array}{ll}
\left(A_{k, T} G_{T, k-1}+B_{k, T} J_{T, k-1}\right) & B_{k, T}\left(\bar{a}_{T}^{-}-\bar{a}_{T}^{+}\right)
\end{array}\right]\left[\begin{array}{c}
\delta \bar{u}_{k-1}^{+} \\
\delta t_{T}
\end{array}\right] .
$$

As in the impulsive Level I method, ${ }^{1}$ the minimum norm solution is used to obtain the desired change in the control variables such that

$$
\left[\begin{array}{c}
\delta \bar{u}_{k-1}^{+} \\
\delta t_{T}
\end{array}\right]=\tilde{M}^{T}\left(\tilde{M} \tilde{M}^{T}\right)^{-1} \delta \bar{R}_{k}
$$

where

$$
\tilde{M}=\left[\left(A_{k, T} G_{T, k-1}+B_{k, T} J_{T, k-1}\right) \quad B_{k, T}\left(\bar{a}_{T}^{-}-\bar{a}_{T}^{+}\right)\right] .
$$

For the first iteration, an initial guess for the duration of the burn is obtained by doing one iteration of an impulsive-maneuver Level I correction and plugging the resulting $\Delta V$ value into the rocket equation. The initial guess for the direction of the burn is taken directly from the $\Delta V$ vector.

\section{B. Level II Process}

The Level II formulation for finite burn maneuvers is not conceptually dissimilar from the impulsive maneuver scheme. ${ }^{1}$ In both cases, the only control variables employed are the positions and times of the patch states. As in the impulsive formulation, a minimum norm solution is used to compute the patch point updates. However, because the dynamical model for the finite burn algorithm is augmented, the partial derivatives are more complex than those in the impulsive maneuver algorithm. The burn is assumed to start with the same initial velocity as the terminal velocity of the preceding arc. Thus, the velocity discontinuity occurs at patch point $k$, the end of the coasting subsegment of the full arc (assuming this arc is then followed by another coast arc). Although this problem at first seems identical to the impulsive maneuver targeting, since the velocity discontinuity falls between two coast arcs, the partial derivatives for $\delta \bar{V}_{k}^{-}$with respect to $\delta \bar{R}_{k-1}$, $t_{k-1}, \delta \bar{R}_{k}$, and $t_{k}$ differ due to the thrust segment at the beginning of the arc.

Extracting $\delta \bar{V}_{k}^{-}$from Equation (2) in the Level 1 formulation section gives

$$
\delta \bar{V}_{k}^{-}=C_{k, T}\left(\delta \bar{R}_{T}-\bar{V}_{T}^{+} \delta t_{T}\right)+D_{k, T}\left(\delta \bar{V}_{T}^{+}-\bar{a}_{T}^{+} \delta t_{T}\right)+\bar{a}_{k}^{-} \delta t_{k} .
$$

Recall from the Level I formulation that $\bar{V}_{T}^{-}=\bar{V}_{T}^{+}$at the terminal point of the burn arc and thus that $\delta \bar{V}_{T}^{+}-\bar{a}_{T}^{+} \delta t_{T}=\delta \bar{V}_{T}^{-}-\bar{a}_{T}^{-} \delta t_{T}+\left(\bar{a}_{T}^{-}-\bar{a}_{T}^{+}\right) \delta t_{T}$. Substituting these expressions into Equation (7) yields

$$
\delta \bar{V}_{k}^{-}=C_{k, T}\left(\delta \bar{R}_{T}-\bar{V}_{T}^{-} \delta t_{T}\right)+D_{k, T}\left[\left(\delta \bar{V}_{T}^{-}-\bar{a}_{T}^{-} \delta t_{T}\right)+\left(\bar{a}_{T}^{-}-\bar{a}_{T}^{+}\right) \delta t_{T}\right]+\bar{a}_{k}^{-} \delta t_{k} .
$$

The first two variational equations embedded within Equation (1) are then summarized as

$$
\begin{aligned}
\left(\delta \bar{R}_{T}-\bar{V}_{T}^{-} \delta t_{T}\right) & =A_{T, k-1}\left(\delta \bar{R}_{k-1}-\bar{V}_{k-1}^{+} \delta t_{k-1}\right)+B_{T, k-1}\left(\delta \bar{V}_{k-1}^{+}-\bar{a}_{k-1}^{+} \delta t_{k-1}\right) \\
& +E_{T, k-1}\left(\delta m_{k-1}^{+}+\dot{m}_{g k-1}^{+} \delta t_{k-1}\right)+F_{T, k-1}\left(\delta \dot{m}_{g k-1}^{+}-\ddot{m}_{g k-1}^{+} \delta t_{k-1}\right) \\
& +G_{T, k-1}\left(\delta \bar{u}_{k-1}^{+}-\dot{\bar{u}}_{k-1}^{+} \delta t_{k-1}\right) \\
\left(\delta \bar{V}_{T}^{-}-\bar{a}_{T}^{-} \delta t_{T}\right) & =C_{T, k-1}\left(\delta \bar{R}_{k-1}-\bar{V}_{k-1}^{+} \delta t_{k-1}\right)+D_{T, k-1}\left(\delta \bar{V}_{k-1}^{+}-\bar{a}_{k-1}^{+} \delta t_{k-1}\right) \\
& +H_{T, k-1}\left(\delta m_{k-1}^{+}+\dot{m}_{g k-1}^{+} \delta t_{k-1}\right)+I_{T, k-1}\left(\delta \dot{m}_{g k-1}^{+}-\ddot{m}_{g k-1}^{+} \delta t_{k-1}\right) \\
& +J_{T, k-1}\left(\delta \bar{u}_{k-1}^{+}-\dot{u}_{k-1}^{+} \delta t_{k-1}\right) .
\end{aligned}
$$

For the Level II process, $\delta m_{k-1}^{+}=\ddot{m}_{g_{k-1}}^{+}=0$ and $\dot{\bar{u}}_{k-1}^{+}=\overline{0}$. It is still assumed that $\dot{m}_{g}$ is a fixed constant, i.e. $\delta \dot{m}_{g k-1}^{+}=0$. Neglecting these zero terms, and substituting the expressions in Equation (9) into Equation (8), an expression is found for $\delta \bar{V}_{k}^{-}$in terms of the state at patch point $k-1$ and the state transition matrix,

$$
\begin{aligned}
\delta \bar{V}_{k}^{-} & =C_{k, T}\left[A_{T, k-1}\left(\delta \bar{R}_{k-1}-\bar{V}_{k-1}^{+} \delta t_{k-1}\right)\right. \\
& \left.+B_{T, k-1}\left(\delta \bar{V}_{k-1}^{+}-\bar{a}_{k-1}^{+} \delta t_{k-1}\right)+E_{T, k-1} \dot{m}_{g_{k-1}}^{+} \delta t_{k-1}+G_{T, k-1} \delta \bar{u}_{k-1}^{+}\right] \\
& +D_{k, T}\left[C_{T, k-1}\left(\delta \bar{R}_{k-1}-\bar{V}_{k-1}^{+} \delta t_{k-1}\right)+D_{T, k-1}\left(\delta \bar{V}_{k-1}^{+}-\bar{a}_{k-1}^{+} \delta t_{k-1}\right)\right. \\
& \left.+H_{T, k-1} \dot{m}_{g_{k-1}}^{+} \delta t_{k-1}+J_{T, k-1} \delta \bar{u}_{k-1}^{+}+\left(\bar{a}_{T}^{-}-\bar{a}_{T}^{+}\right) \delta t_{T}\right]+\bar{a}_{k}^{-} \delta t_{k} .
\end{aligned}
$$


In order to write $\delta \bar{V}_{k}^{-}$only in terms of the Level II control variables, the first vector equation from Equation (1) is used to solve for $\delta \bar{V}_{k-1}^{+}, \delta \bar{u}_{k-1}^{+}$, and $\delta t_{T}$. Substituting for $\bar{V}_{T}^{+}$and $\delta \bar{V}_{T}^{+}-\bar{a}_{T}^{+} \delta t_{T}$ as shown previously, this equation is

$$
\delta \bar{R}_{k}-\bar{V}_{k}^{-} \delta t_{k}=A_{k, T}\left(\delta \bar{R}_{T}-\bar{V}_{T}^{-} \delta t_{T}\right)+B_{k, T}\left[\left(\delta \bar{V}_{T}^{-}-\bar{a}_{T}^{-} \delta t_{T}\right)+\left(\bar{a}_{T}^{-}-\bar{a}_{T}^{+}\right) \delta t_{T}\right] .
$$

Dropping the terms that are equal to zero and substituting the same expressions from Equation (9) that were used to form Equation (10), this first vector equation becomes

$$
\begin{aligned}
\delta \bar{R}_{k}-\bar{V}_{k}^{-} \delta t_{k} & =A_{k, T}\left[A_{T, k-1}\left(\delta \bar{R}_{k-1}-\bar{V}_{k-1}^{+} \delta t_{k-1}\right)+B_{T, k-1}\left(\delta \bar{V}_{k-1}^{+}-\bar{a}_{k-1}^{+} \delta t_{k-1}\right)\right. \\
& \left.+E_{T, k-1} \dot{m}_{g_{k-1}}^{+} \delta t_{k-1}+G_{T, k-1} \delta \bar{u}_{k-1}^{+}\right] \\
& +B_{k, T}\left[C_{T, k-1}\left(\delta \bar{R}_{k-1}-\bar{V}_{k-1}^{+} \delta t_{k-1}\right)\right. \\
& +D_{T, k-1}\left(\delta \bar{V}_{k-1}^{+}-\bar{a}_{k-1}^{+} \delta t_{k-1}\right)+H_{T, k-1} \dot{m}_{g_{k-1}}^{+} \delta t_{k-1} \\
& \left.+J_{T, k-1} \delta \bar{u}_{k-1}^{+}+\left(\bar{a}_{T}^{-}-\bar{a}_{T}^{+}\right) \delta t_{T}\right] .
\end{aligned}
$$

Further rearranging Equation (12) into vector form to isolate $\delta \bar{V}_{k-1}^{+}, \delta \bar{u}_{k-1}^{+}$, and $\delta t_{T}$ yields

$$
\begin{aligned}
& \delta \bar{R}_{k}-\bar{V}_{k}^{-} \delta t_{k}-\left(A_{k, T} A_{T, k-1}+B_{k, T} C_{T, k-1}\right)\left(\delta \bar{R}_{k-1}-\bar{V}_{k-1}^{+} \delta t_{k-1}\right)+ \\
& \left(\begin{array}{lll}
\left.A_{k, T} B_{T, k-1}+B_{k, T} D_{T, k-1}\right) \bar{a}_{k-1}^{+} \delta t_{k-1}-\left(A_{k, T} E_{T, k-1}+B_{k, T} H_{T, k-1}\right) \dot{m}_{g_{k-1}}^{+} \delta t_{k-1} \\
=\left[\begin{array}{lll}
\left(A_{k, T} B_{T, k-1}+B_{k, T} D_{T, k-1}\right) & \left(A_{k, T} G_{T, k-1}+B_{k, T} J_{T, k-1}\right) & B_{k, T}\left(\bar{a}_{T}^{-}-\bar{a}_{T}^{+}\right)
\end{array}\right]\left[\begin{array}{c}
\delta \bar{V}_{k-1}^{+} \\
\delta \bar{u}_{k-1}^{+} \\
\delta t_{T}
\end{array}\right] .
\end{array}\right.
\end{aligned}
$$

The minimum norm solution is used to solve Equation (13) for $\left[\begin{array}{lll}\delta \bar{V}_{k-1}^{+} & \delta \bar{u}_{k-1}^{+} & \delta t_{T}\end{array}\right]^{T}$ in terms of the Level 2 control variables such that

$$
\begin{aligned}
{\left[\begin{array}{c}
\delta \bar{V}_{k-1}^{+} \\
\delta \bar{u}_{k-1}^{+} \\
\delta t_{T}
\end{array}\right] } & =Z^{T}\left(Z Z^{T}\right)^{-1}\left[\delta \bar{R}_{k}-\bar{V}_{k}^{-} \delta t_{k}\right. \\
& -\left(A_{k, T} A_{T, k-1}+B_{k, T} C_{T, k-1}\right)\left(\delta \bar{R}_{k-1}-\bar{V}_{k-1}^{+} \delta t_{k-1}\right) \\
& +\left(A_{k, T} B_{T, k-1}+B_{k, T} D_{T, k-1}\right) \bar{a}_{k-1}^{+} \delta t_{k-1} \\
& \left.-\left(A_{k, T} E_{T, k-1}+B_{k, T} H_{T, k-1}\right) \dot{m}_{g_{k-1}}^{+} \delta t_{k-1}\right] .
\end{aligned}
$$

Here, $Z=\left[\begin{array}{lll}\left(A_{k, T} B_{T, k-1}+B_{k, T} D_{T, k-1}\right) & \left(A_{k, T} G_{T, k-1}+B_{k, T} J_{T, k-1}\right) & B_{k, T}\left(\bar{a}_{T}^{-}-\bar{a}_{T}^{+}\right)\end{array}\right]$. Going back to Equation (10), this expression is rewritten in terms of the Level 2 controls and the vector $\left[\begin{array}{lll}\delta \bar{V}_{k-1}^{+} & \delta \bar{u}_{k-1}^{+} & \delta t_{T}\end{array}\right]^{T}$ as follows

$$
\begin{aligned}
\delta \bar{V}_{k}^{-} & =\bar{a}_{k}^{-} \delta t_{k}+\left(C_{k, T} A_{T, k-1}+D_{k, T} C_{T, k-1}\right)\left(\delta \bar{R}_{k-1}-\bar{V}_{k-1}^{+} \delta t_{k-1}\right) \\
& -\left(C_{k, T} B_{T, k-1}+D_{k, T} D_{T, k-1}\right) \bar{a}_{k-1}^{+} \delta t_{k-1}+\left(C_{k, T} E_{T, k-1}+D_{k, T} H_{T, k-1}\right) \dot{m}_{g_{k-1}}^{+} \delta t_{k-1} \\
& +\left[\begin{array}{lll}
\left(C_{k, T} B_{T, k-1}+D_{k, T} D_{T, k-1}\right) & \left(C_{k, T} G_{T, k-1}+D_{k, T} J_{T, k-1}\right) & D_{k, T}\left(\bar{a}_{T}^{-}-\bar{a}_{T}^{+}\right)
\end{array}\right]\left[\begin{array}{c}
\delta \bar{V}_{k-1}^{+} \\
\delta \bar{u}_{k-1}^{+} \\
\delta t_{T}
\end{array}\right]
\end{aligned}
$$

Substituting the expression for $\left[\begin{array}{lll}\delta \bar{V}_{k-1}^{+} & \delta \bar{u}_{k-1}^{+} & \delta t_{T}\end{array}\right]^{T}$ into Equation (15) gives

$$
\begin{aligned}
& \delta \bar{V}_{k}^{-}=\bar{a}_{k}^{-} \delta t_{k}+\left(C_{k, T} A_{T, k-1}+D_{k, T} C_{T, k-1}\right)\left(\delta \bar{R}_{k-1}-\bar{V}_{k-1}^{+} \delta t_{k-1}\right) \\
& -\left(C_{k, T} B_{T, k-1}+D_{k, T} D_{T, k-1}\right) \bar{a}_{k-1}^{+} \delta t_{k-1}+\left(C_{k, T} E_{T, k-1}+D_{k, T} H_{T, k-1}\right) \dot{m}_{g_{k-1}}^{+} \delta t_{k-1} \\
& +\left[\begin{array}{lll}
\left(C_{k, T} B_{T, k-1}+D_{k, T} D_{T, k-1}\right) & \left(C_{k, T} G_{T, k-1}+D_{k, T} J_{T, k-1}\right) & D_{k, T}\left(\bar{a}_{T}^{-}-\bar{a}_{T}^{+}\right)
\end{array}\right] \\
& * Z^{T}\left(Z Z^{T}\right)^{-1}\left[\delta \bar{R}_{k}-\bar{V}_{k}^{-} \delta t_{k}-\left(A_{k, T} A_{T, k-1}+B_{k, T} C_{T, k-1}\right)\left(\delta \bar{R}_{k-1}-\bar{V}_{k-1}^{+} \delta t_{k-1}\right)\right. \\
& \left.+\left(A_{k, T} B_{T, k-1}+B_{k, T} D_{T, k-1}\right) \bar{a}_{k-1}^{+} \delta t_{k-1}-\left(A_{k, T} E_{T, k-1}+B_{k, T} H_{T, k-1}\right) \dot{m}_{g_{k-1}}^{+} \delta t_{k-1}\right] \text {. }
\end{aligned}
$$


Let

$$
\tilde{Z}=\left[\begin{array}{lll}
\left(C_{k, T} B_{T, k-1}+D_{k, T} D_{T, k-1}\right) & \left(C_{k, T} G_{T, k-1}+D_{k, T} J_{T, k-1}\right) & D_{k, T}\left(\bar{a}_{T}^{-}-\bar{a}_{T}^{+}\right)
\end{array}\right] Z^{T}\left(Z Z^{T}\right)^{-1} .
$$

Then, rearranging Equation (16) to isolate the control variables yields

$$
\begin{aligned}
\delta \bar{V}_{k}^{-} & =\left[\left(C_{k, T} A_{T, k-1}+D_{k, T} C_{T, k-1}\right)-\tilde{Z}\left(A_{k, T} A_{T, k-1}+B_{k, T} C_{T, k-1}\right)\right] \delta \bar{R}_{k-1} \\
& +\left(\left[\left(C_{k, T} E_{T, k-1}+D_{k, T} H_{T, k-1}\right)-\tilde{Z}\left(A_{k, T} E_{T, k-1}+B_{k, T} H_{T, k-1}\right)\right] \dot{m}_{g_{k-1}}^{+}\right. \\
& -\left[\left(C_{k, T} B_{T, k-1}+D_{k, T} D_{T, k-1}\right)-\tilde{Z}\left(A_{k, T} B_{T, k-1}+B_{k, T} D_{T, k-1}\right)\right] \bar{a}_{k-1}^{+} \\
& \left.-\left[\left(C_{k, T} A_{T, k-1}+D_{k, T} C_{T, k-1}\right)-\tilde{Z}\left(A_{k, T} A_{T, k-1}+B_{k, T} C_{T, k-1}\right)\right] \bar{V}_{k-1}^{+}\right) \delta t_{k-1} \\
& +\tilde{Z} \delta \bar{R}_{k}+\left(\bar{a}_{k}^{-}-\tilde{Z} \bar{V}_{k}^{-}\right) \delta t_{k} .
\end{aligned}
$$

Thus, the partials of $\delta \bar{V}_{k}^{-}$are

$$
\begin{aligned}
\frac{\partial \delta \bar{V}_{k}^{-}}{\partial \delta \bar{R}_{k-1}} & =\left(C_{k, T} A_{T, k-1}+D_{k, T} C_{T, k-1}\right)-\tilde{Z}\left(A_{k, T} A_{T, k-1}+B_{k, T} C_{T, k-1}\right) \\
\frac{\partial \delta \bar{V}_{k}^{-}}{\partial \delta t_{k-1}}= & {\left[\left(C_{k, T} E_{T, k-1}+D_{k, T} H_{T, k-1}\right)-\tilde{Z}\left(A_{k, T} E_{T, k-1}+B_{k, T} H_{T, k-1}\right)\right] \dot{m}_{g_{k-1}}^{+} } \\
- & {\left[\left(C_{k, T} B_{T, k-1}+D_{k, T} D_{T, k-1}\right)-\tilde{Z}\left(A_{k, T} B_{T, k-1}+B_{k, T} D_{T, k-1}\right)\right] \bar{a}_{k-1}^{+} } \\
& -\left[\left(C_{k, T} A_{T, k-1}+D_{k, T} C_{T, k-1}\right)-\tilde{Z}\left(A_{k, T} A_{T, k-1}+B_{k, T} C_{T, k-1}\right)\right] \bar{V}_{k-1}^{+} \\
\frac{\partial \delta \bar{V}_{k}^{-}}{\partial \delta \bar{R}_{k}} & =\tilde{Z} \\
\frac{\partial \delta \bar{V}_{k}^{-}}{\partial \delta t_{k}} & =\left(\bar{a}_{k}^{-}-\tilde{Z} \bar{V}_{k}^{-}\right) .
\end{aligned}
$$

Because it is assumed that the arc from patch points $k$ to $k+1$ is a coast arc, the partial derivatives of $\delta \bar{V}_{k}^{+}$ are the same as for the impulsive case. The partial derivatives of $\Delta \bar{V}_{k}$ are therefore

$$
\begin{aligned}
\frac{\partial \Delta \bar{V}_{k}}{\partial \bar{R}_{k-1}}= & -\left(C_{k, T} A_{T, k-1}+D_{k, T} C_{T, k-1}\right)-\tilde{Z}\left(A_{k, T} A_{T, k-1}+B_{k, T} C_{T, k-1}\right) \\
\frac{\partial \Delta \bar{V}_{k}}{\partial t_{k-1}}= & -\left(\left[\left(C_{k, T} E_{T, k-1}+D_{k, T} H_{T, k-1}\right)-\tilde{Z}\left(A_{k, T} E_{T, k-1}+B_{k, T} H_{T, k-1}\right)\right] \dot{m}_{g_{k-1}}^{+}\right. \\
& -\left[\left(C_{k, T} B_{T, k-1}+D_{k, T} D_{T, k-1}\right)-\tilde{Z}\left(A_{k, T} B_{T, k-1}+B_{k, T} D_{T, k-1}\right)\right] \bar{a}_{k-1}^{+} \\
& \left.-\left[\left(C_{k, T} A_{T, k-1}+D_{k, T} C_{T, k-1}\right)-\tilde{Z}\left(A_{k, T} A_{T, k-1}+B_{k, T} C_{T, k-1}\right)\right] \bar{V}_{k-1}^{+}\right) \\
\frac{\partial \Delta \bar{V}_{k}}{\partial \bar{R}_{k}}= & -B_{k+1, k}^{-1} A_{k+1, k}-\tilde{Z} \\
\frac{\partial \Delta \bar{V}_{k}}{\partial t_{k}}= & B_{k+1, k}^{-1} A_{k+1, k} \bar{V}_{k}^{+}+\bar{a}_{k}^{+}-\left(\bar{a}_{k}^{-}-\tilde{Z} \bar{V}_{k}^{-}\right) \\
\frac{\partial \Delta \bar{V}_{k}}{\partial \bar{R}_{k+1}}= & B_{k+1, k}^{-1}, \\
\frac{\partial \Delta \bar{V}_{k}}{\partial t_{k+1}}= & -B_{k+1, k}^{-1} \bar{V}_{k+1}^{-} .
\end{aligned}
$$

The desired changes to the control variables are found using the minimum norm solution,

$$
\left[\begin{array}{c}
\delta \bar{R}_{k-1} \\
\delta t_{k-1} \\
\delta \bar{R}_{k} \\
\delta t_{k} \\
\delta \bar{R}_{k+1} \\
\delta t_{k+1}
\end{array}\right]=M^{T}\left(M M^{T}\right)^{-1} \Delta \bar{V}_{k}
$$

where $M=\left[\begin{array}{llllll}\frac{\partial \Delta \bar{V}_{k}}{\partial \bar{R}_{k-1}} & \frac{\partial \Delta \bar{V}_{k}}{\partial t_{k-1}} & \frac{\partial \Delta \bar{V}_{k}}{\partial \bar{R}_{k}} & \frac{\partial \Delta \bar{V}_{k}}{\partial t_{k}} & \frac{\partial \Delta \bar{V}_{k}}{\partial \bar{R}_{k+1}} & \frac{\partial \Delta \bar{V}_{k}}{\partial t_{k+1}}\end{array}\right]$. 


\section{Maneuver Sum Constraint}

In addition to the velocity continuity constraint, several trajectory constraints are imposed in the Level II process. ${ }^{2}$ One such constraint is on the total $\Delta V$ sum of the maneuvers. The finite burn formulation of this constraint is based on the impulsive maneuver sum constraint, ${ }^{3}$ and in fact uses the exact same approach to place the relevant partial derivatives into the $M$ matrix. Only the composition of those partial derivatives and the error calculation process change.

Denote the patch point where the burn occurs as patch point $k$, rather than $k-1$ as in the previous sections. This is so that the Level II control variables for this constraint are still $\bar{R}_{k-1}, t_{k-1}, \bar{R}_{k}, t_{k}, \bar{R}_{k+1}$, and $t_{k+1}$, as is the convention for this process. To derive the burn maneuver constraint, it is necessary to determine the partial derivatives of the magnitude of $\Delta \bar{V}_{k}$, the $\Delta V$ resulting from the burn maneuver at patch point $k$, with respect to the Level II control variables. From the rocket equation, $\Delta V_{k}$ is given by

$$
\Delta V_{k}=-I_{s p} g_{0} \ln \left(1-\frac{\dot{m}_{g_{k}} \Delta t_{\text {burn }}}{m_{k}}\right),
$$

where $\Delta t_{\text {burn }}=t_{T}-t_{k}$. The partial derivative of $\Delta V_{k}$ with respect to the burn duration at patch point $k$ is given by

$$
\frac{\partial \Delta V_{k}}{\partial \Delta t_{b u r n}}=I_{s p} g_{0}\left(\frac{m_{k}}{m_{k}-\dot{m}_{g_{k}} \Delta t_{b u r n}}\right)\left(\frac{\dot{m}_{g_{k}}}{m_{k}}\right) .
$$

Next, the partial derivatives of $\Delta t_{\text {burn }}$ with respect to the control variables are necessary. From the definition of $\Delta t_{b u r n}$, it is evident that an expression for $\delta t_{T}$ in terms of the control variables is required. This is determined using the variational equations from points $k-1$ to $k, k$ to $T$ (the termination of the burn segment), and $k+1$ to $T$;

$$
\begin{gathered}
{\left[\begin{array}{c}
\delta \bar{R}_{k}-\bar{V}_{k}^{-} \delta t_{k} \\
\delta \bar{V}_{k}^{-}-\bar{a}_{k}^{-} \delta t_{k}
\end{array}\right]=\left[\begin{array}{lll}
A_{k, k-1} & B_{k, k-1} \\
C_{k, k-1} & D_{k, k-1}
\end{array}\right]\left[\begin{array}{c}
\delta \bar{R}_{k-1}-\bar{V}_{k-1}^{+} \delta t_{k-1} \\
\delta \bar{V}_{k-1}^{+}-\bar{a}_{k-1}^{+} \delta t_{k-1}
\end{array}\right],} \\
{\left[\begin{array}{c}
\delta \bar{R}_{T}-\bar{V}_{T}^{-} \delta t_{T} \\
\delta \bar{V}_{T}^{-}-\bar{a}_{T}^{-} \delta t_{T} \\
\delta m_{T}^{-}+\dot{m}_{g_{T}}^{-} \delta t_{T} \\
\delta \dot{m}_{g_{T}}^{-}-\ddot{m}_{g_{T}}^{-} \delta t_{T} \\
\delta \bar{u}_{T}^{-}-\dot{\bar{u}}_{T}^{-} \delta t_{T}
\end{array}\right]=\left[\begin{array}{ccccc}
A_{T, k} & B_{T, k} & E_{T, k} & F_{T, k} & G_{T, k} \\
C_{T, k} & D_{T, k} & H_{T, k} & I_{T, k} & J_{T, k} \\
K_{T, k} & L_{T, k} & M_{T, k} & N_{T, k} & O_{T, k} \\
P_{T, k} & Q_{T, k} & R_{T, k} & S_{T, k} & T_{T, k} \\
U_{T, k} & V_{T, k} & W_{T, k} & X_{T, k} & Y_{T, k}
\end{array}\right]\left[\begin{array}{c}
\delta \bar{R}_{k}-\bar{V}_{k}^{+} \delta t_{k} \\
\delta \bar{V}_{k}^{+}-\bar{a}_{k}^{+} \delta t_{k} \\
\delta m_{k}^{+}+\dot{m}_{g_{k}}^{+} \delta t_{k} \\
\delta \dot{m}_{g_{k}}^{+}-\ddot{m}_{g_{k}}^{+} \delta t_{k} \\
\delta \bar{u}_{k}^{+}-\dot{\bar{u}}_{k}^{+} \delta t_{k}
\end{array}\right],}
\end{gathered}
$$

and

$$
\left[\begin{array}{c}
\delta \bar{R}_{T}-\bar{V}_{T}^{+} \delta t_{T} \\
\delta \bar{V}_{T}^{+}-\bar{a}_{T}^{+} \delta t_{T}
\end{array}\right]=\left[\begin{array}{cc}
A_{T, k+1} & B_{T, k+1} \\
C_{T, k+1} & D_{T, k+1}
\end{array}\right]\left[\begin{array}{c}
\delta \bar{R}_{k+1}-\bar{V}_{k+1}^{-} \delta t_{k+1} \\
\delta \bar{V}_{k+1}^{-}-\bar{a}_{k+1}^{-} \delta t_{k+1}
\end{array}\right] .
$$

Recall that $\bar{V}_{T}^{+}=\bar{V}_{T}^{-}$, and therefore $\delta \bar{V}_{T}^{+}=\delta \bar{V}_{T}^{-}$. Then the second vector equation of Equation (25) is subtracted from the second vector equation of Equation (26) to isolate $\delta t_{T}$

$$
\begin{aligned}
\left(\bar{a}_{T}^{-}-\bar{a}_{T}^{+}\right) \delta t_{T} & =-C_{T, k}\left(\delta \bar{R}_{k}-\bar{V}_{k}^{+} \delta t_{k}\right)-D_{T, k}\left(\delta \bar{V}_{k}^{+}-\bar{a}_{k}^{+} \delta t_{k}\right) \\
& -H_{T, k}\left(\delta m_{k}+\dot{m}_{g_{k}}^{+} \delta t_{k}\right)-J_{T, k} \delta \bar{u}_{k}^{+}+C_{T, k+1}\left(\delta \bar{R}_{k+1}-\bar{V}_{k+1}^{-} \delta t_{k+1}\right) \\
& +D_{T, k+1}\left(\delta \bar{V}_{k+1}^{-}-\bar{a}_{k+1}^{-} \delta t_{k+1}\right) .
\end{aligned}
$$

Since there is no velocity discontinuity at patch point $k, \delta \bar{V}_{k}^{+}=\delta \bar{V}_{k}^{-}$; thus, from Equation (24),

$$
\begin{aligned}
\delta \bar{V}_{k}^{+}-\bar{a}_{k}^{+} \delta t_{k} & =\left(\delta \bar{V}_{k}^{-}-\bar{a}_{k}^{-} \delta t_{k}\right)+\left(\bar{a}_{k}^{-}-\bar{a}_{k}^{+}\right) \\
& =C_{k, k-1}\left(\delta \bar{R}_{k-1}-\bar{V}_{k-1}^{+} \delta t_{k-1}\right)+D_{k, k-1}\left(\delta \bar{V}_{k-1}^{+}-\bar{a}_{k-1}^{+} \delta t_{k-1}\right)+\left(\bar{a}_{k}^{-}-\bar{a}_{k}^{+}\right) \delta t_{k} .
\end{aligned}
$$

Substituting this expression back into Equation (27) yields

$$
\begin{aligned}
\left(\bar{a}_{T}^{-}-\bar{a}_{T}^{+}\right) \delta t_{T} & =-C_{T, k}\left(\delta \bar{R}_{k}-\bar{V}_{k}^{+} \delta t_{k}\right)-D_{T, k}\left[C_{k, k-1}\left(\delta \bar{R}_{k-1}-\bar{V}_{k-1}^{+} \delta t_{k-1}\right)\right. \\
& \left.+D_{k, k-1}\left(\delta \bar{V}_{k-1}^{+}-\bar{a}_{k-1}^{+} \delta t_{k-1}\right)+\left(\bar{a}_{k}^{-}-\bar{a}_{k}^{+}\right)\right]-H_{T, k}\left(\delta m_{k}+\dot{m}_{g_{k}}^{+} \delta t_{k}\right) \\
& -J_{T, k} \delta \bar{u}_{k}^{+}+C_{T, k+1}\left(\delta \bar{R}_{k+1}-\bar{V}_{k+1}^{-} \delta t_{k+1}\right) \\
& +D_{T, k+1}\left(\delta \bar{V}_{k+1}^{-}-\bar{a}_{k+1}^{-} \delta t_{k+1}\right) .
\end{aligned}
$$


The term $\delta \bar{V}_{k-1}^{+}-\bar{a}_{k-1}^{+} \delta t_{k-1}$ may be written in terms of the control variables by rearranging the first variational equation of Equation (24),

$$
\delta \bar{V}_{k-1}^{+}-\bar{a}_{k-1}^{+} \delta t_{k-1}=B_{k, k-1}^{-1}\left[\left(\delta \bar{R}_{k}-\bar{V}_{k}^{-} \delta t_{k}\right)-A_{k, k-1}\left(\delta \bar{R}_{k-1}-\bar{V}_{k-1}^{+} \delta t_{k}\right)\right] .
$$

Thus, Equation (29) may also be written as

$$
\begin{aligned}
\left(\bar{a}_{T}^{-}-\bar{a}_{T}^{+}\right) \delta t_{T} & =-C_{T, k}\left(\delta \bar{R}_{k}-\bar{V}_{k}^{+} \delta t_{k}\right)-D_{T, k}\left[C_{k, k-1}\left(\delta \bar{R}_{k-1}-\bar{V}_{k-1}^{+} \delta t_{k-1}\right)\right. \\
& \left.+D_{k, k-1} B_{k, k-1}^{-1}\left[\left(\delta \bar{R}_{k}-\bar{V}_{k}^{-} \delta t_{k}\right)-A_{k, k-1}\left(\delta \bar{R}_{k-1}-\bar{V}_{k-1}^{+} \delta t_{k}\right)\right]+\left(\bar{a}_{k}^{-}-\bar{a}_{k}^{+}\right) \delta t_{k}\right] \\
& -H_{T, k}\left(\delta m_{k}+\dot{m}_{g_{k}}^{+} \delta t_{k}\right)-J_{T, k} \delta \bar{u}_{k}^{+}+C_{T, k+1}\left(\delta \bar{R}_{k+1}-\bar{V}_{k+1}^{-} \delta t_{k+1}\right) \\
& +D_{T, k+1}\left(\delta \bar{V}_{k+1}^{-}-\bar{a}_{k+1}^{-} \delta t_{k+1}\right) .
\end{aligned}
$$

Grouping like terms and rearranging to combine the non-control variables into a single vector yields

$$
\begin{aligned}
\left(\bar{a}_{T}^{-}-\bar{a}_{T}^{+}\right) \delta t_{T} & =-\left(C_{T, k}+D_{T, k} D_{k, k-1} B_{k, k-1}^{-1}\right)\left(\delta \bar{R}_{k}-\bar{V}_{k}^{+} \delta t_{k}\right) \\
& -D_{T, k}\left(C_{k, k-1}-D_{k, k-1} B_{k, k-1}^{-1} A_{k, k-1}\right)\left(\delta \bar{R}_{k-1}-\bar{V}_{k-1}^{+} \delta t_{k-1}\right) \\
& -D_{T, k}\left(\bar{a}_{k}^{-}-\bar{a}_{k}^{+}\right) \delta t_{k}-H_{T, k}\left(\delta m_{k}+\dot{m}_{g_{k}}^{+} \delta t_{k}\right) \\
& +C_{T, k+1}\left(\delta \bar{R}_{k+1}-\bar{V}_{k+1}^{-} \delta t_{k+1}\right)-D_{T, k+1} \bar{a}_{k+1}^{-} \delta t_{k+1} \\
& +\left[\begin{array}{ll}
-J_{T, k} & D_{T, k+1}
\end{array}\right]\left[\begin{array}{c}
\delta \bar{u}_{k}^{+} \\
\delta \bar{V}_{k+1}^{-}
\end{array}\right] .
\end{aligned}
$$

It has been shown, in the derivation of impulsive targeting algorithm, that $\left(C_{k, k-1}-D_{k, k-1} B_{k, k-1}^{-1} A_{k, k-1}\right)=$ $B_{k-1, k}^{-1} ;$ thus, Equation (32) can be somewhat reduced to

$$
\begin{aligned}
\left(\bar{a}_{T}^{-}-\bar{a}_{T}^{+}\right) \delta t_{T} & =-\left(C_{T, k}+D_{T, k} D_{k, k-1} B_{k, k-1}^{-1}\right)\left(\delta \bar{R}_{k}-\bar{V}_{k}^{+} \delta t_{k}\right) \\
& -D_{T, k} B_{k-1, k}^{-1}\left(\delta \bar{R}_{k-1}-\bar{V}_{k-1}^{+} \delta t_{k-1}\right) \\
& -D_{T, k}\left(\bar{a}_{k}^{-}-\bar{a}_{k}^{+}\right) \delta t_{k}-H_{T, k}\left(\delta m_{k}+\dot{m}_{g_{k}}^{+} \delta t_{k}\right) \\
& +C_{T, k+1}\left(\delta \bar{R}_{k+1}-\bar{V}_{k+1}^{-} \delta t_{k+1}\right)-D_{T, k+1} \bar{a}_{k+1}^{-} \delta t_{k+1} \\
& +\left[\begin{array}{ll}
-J_{T, k} & D_{T, k+1}
\end{array}\right]\left[\begin{array}{c}
\delta \bar{u}_{k}^{+} \\
\delta \bar{V}_{k+1}^{-}
\end{array}\right]
\end{aligned}
$$

In order to express $\left[\begin{array}{cc}\delta \bar{u}_{k}^{+} & \delta \bar{V}_{k+1}^{-}\end{array}\right]^{T}$ in terms of the control variables, the first vector equations from Equation (25) and Equation (26) are used. Because $\bar{V}_{T}^{+}=\bar{V}_{T}^{-}$, it is also true that $\delta \bar{R}_{T}-\bar{V}_{T}^{+}=\delta \bar{R}_{T}-\delta \bar{V}_{T}^{-}$. Thus,

$$
\begin{aligned}
& A_{T, k}\left(\delta \bar{R}_{k}-\bar{V}_{k}^{+} \delta t_{k}\right)+B_{T, k}\left(\delta \bar{V}_{k}^{+}-\bar{a}_{k}^{+} \delta t_{k}\right)+E_{T, k}\left(\delta m_{k}+\dot{m}_{g_{k}}^{+} \delta t_{k}\right)+G_{T, k} \delta \bar{u}_{k}^{+} \\
& =A_{T, k+1}\left(\delta \bar{R}_{k+1}-\bar{V}_{k+1}^{-} \delta t_{k+1}\right)+B_{T, k+1}\left(\delta \bar{V}_{k+1}^{-}-\bar{a}_{k+1}^{-} \delta t_{k+1}\right) .
\end{aligned}
$$

Using the relationship in Equation (30), the previous equation may be rewritten as

$$
\begin{aligned}
& A_{T, k}\left(\delta \bar{R}_{k}-\bar{V}_{k}^{+} \delta t_{k}\right)+B_{T, k}\left[C_{k, k-1}\left(\delta \bar{R}_{k-1}-\bar{V}_{k-1}^{+} \delta t_{k-1}\right)\right. \\
& \left.+D_{k, k-1} B_{k, k-1}^{-1}\left[\left(\delta \bar{R}_{k}-\bar{V}_{k}^{-} \delta t_{k}\right)-A_{k, k-1}\left(\delta \bar{R}_{k-1}-\bar{V}_{k-1}^{+} \delta t_{k}\right)\right]+\left(\bar{a}_{k}^{-}-\bar{a}_{k}^{+}\right) \delta t_{k}\right] \\
& +E_{T, k}\left(\delta m_{k}+\dot{m}_{g_{k}}^{+} \delta t_{k}\right)+G_{T, k} \delta \bar{u}_{k}^{+} \\
& =A_{T, k+1}\left(\delta \bar{R}_{k+1}-\bar{V}_{k+1}^{-} \delta t_{k+1}\right)+B_{T, k+1}\left(\delta \bar{V}_{k+1}^{-}-\bar{a}_{k+1}^{-} \delta t_{k+1}\right) .
\end{aligned}
$$

Rearranging this equation yields

$$
\begin{aligned}
& \left(A_{T, k}+B_{T, k} D_{k, k-1} B_{k, k-1}^{-1}\right)\left(\delta \bar{R}_{k}-\bar{V}_{k}^{-} \delta t_{k}\right)+B_{T, k}\left(C_{k, k-1}\right. \\
& \left.-D_{k, k-1} B_{k, k-1}^{-1} A_{k, k-1}\right)\left(\delta \bar{R}_{k-1}-\bar{V}_{k-1}^{+} \delta t_{k}\right) \\
& +B_{T, k}\left(\bar{a}_{k}^{-}-\bar{a}_{k}^{+}\right) \delta t_{k}+E_{T, k}\left(\delta m_{k}+\dot{m}_{g_{k}}^{+} \delta t_{k}\right) \\
& -A_{T, k+1}\left(\delta \bar{R}_{k+1}-\bar{V}_{k+1}^{-} \delta t_{k+1}\right)+B_{T, k+1} \bar{a}_{k+1}^{-} \delta t_{k+1} \\
& =\left[\begin{array}{ll}
-G_{T, k} & B_{T, k+1}
\end{array}\right]\left[\begin{array}{c}
\delta \bar{u}_{k}^{+} \\
\delta \bar{V}_{k+1}^{-}
\end{array}\right] .
\end{aligned}
$$


Again using the relationship $\left(C_{k, k-1}-D_{k, k-1} B_{k, k-1}^{-1} A_{k, k-1}\right)=B_{k-1, k}^{-1}$, Equation (36) becomes

$$
\begin{aligned}
& \left(A_{T, k}+B_{T, k} D_{k, k-1} B_{k, k-1}^{-1}\right)\left(\delta \bar{R}_{k}-\bar{V}_{k}^{-} \delta t_{k}\right)+B_{T, k} B_{k-1, k}^{-1}\left(\delta \bar{R}_{k-1}-\bar{V}_{k-1}^{+} \delta t_{k}\right) \\
& +B_{T, k}\left(\bar{a}_{k}^{-}-\bar{a}_{k}^{+}\right) \delta t_{k}+E_{T, k}\left(\delta m_{k}+\dot{m}_{g_{k}}^{+} \delta t_{k}\right) \\
& -A_{T, k+1}\left(\delta \bar{R}_{k+1}-\bar{V}_{k+1}^{-} \delta t_{k+1}\right)+B_{T, k+1} \bar{a}_{k+1}^{-} \delta t_{k+1} \\
& =\left[\begin{array}{ll}
-G_{T, k} & B_{T, k+1}
\end{array}\right]\left[\begin{array}{c}
\delta \bar{u}_{k}^{+} \\
\delta \bar{V}_{k+1}^{-}
\end{array}\right] .
\end{aligned}
$$

Letting $S=\left[\begin{array}{ll}-G_{T, k} & B_{T, k+1}\end{array}\right]$, the minimum norm solution to Equation (37) gives an expression for $\left[\begin{array}{ll}\delta \bar{u}_{k}^{+} & \delta \bar{V}_{k+1}^{-}\end{array}\right]^{T}$ in terms of the control variables:

$$
\begin{aligned}
{\left[\begin{array}{c}
\delta \bar{u}_{k}^{+} \\
\delta \bar{V}_{k+1}^{-}
\end{array}\right] } & =S^{T}\left(S S^{T}\right)^{-1}\left[\left(A_{T, k}+B_{T, k} D_{k, k-1} B_{k, k-1}^{-1}\right)\left(\delta \bar{R}_{k}-\bar{V}_{k}^{-} \delta t_{k}\right)\right. \\
& +B_{T, k} B_{k-1, k}^{-1}\left(\delta \bar{R}_{k-1}-\bar{V}_{k-1}^{+} \delta t_{k}\right)+B_{T, k}\left(\bar{a}_{k}^{-}-\bar{a}_{k}^{+}\right) \delta t_{k} \\
& +E_{T, k}\left(\delta m_{k}+\dot{m}_{g_{k}}^{+} \delta t_{k}\right)-A_{T, k+1}\left(\delta \bar{R}_{k+1}-\bar{V}_{k+1}^{-} \delta t_{k+1}\right) \\
& \left.+B_{T, k+1} \bar{a}_{k+1}^{-} \delta t_{k+1}\right] .
\end{aligned}
$$

This can be substituted back into Equation (33) to obtain an expression for $\delta t_{T}$ in terms of the control variables. Let $\tilde{S}=\left[\begin{array}{ll}-J_{T, k} & D_{T, k+1}\end{array}\right] S^{T}\left(S S^{T}\right)^{-1}$; Equation (33) can then be written as

$$
\begin{aligned}
\left(\bar{a}_{T}^{-}-\bar{a}_{T}^{+}\right) \delta t_{T} & =-\left(C_{T, k}+D_{T, k} D_{k, k-1} B_{k, k-1}^{-1}\right)\left(\delta \bar{R}_{k}-\bar{V}_{k}^{+} \delta t_{k}\right) \\
& -D_{T, k} B_{k-1, k}^{-1}\left(\delta \bar{R}_{k-1}-\bar{V}_{k-1}^{+} \delta t_{k-1}\right)-D_{T, k}\left(\bar{a}_{k}^{-}-\bar{a}_{k}^{+}\right) \delta t_{k} \\
& -H_{T, k}\left(\delta m_{k}+\dot{m}_{g_{k}}^{+} \delta t_{k}\right)+C_{T, k+1}\left(\delta \bar{R}_{k+1}-\bar{V}_{k+1}^{-} \delta t_{k+1}\right) \\
& -D_{T, k+1} \bar{a}_{k+1}^{-} \delta t_{k+1} \\
& +\tilde{S}\left[\left(A_{T, k}+B_{T, k} D_{k, k-1} B_{k, k-1}^{-1}\right)\left(\delta \bar{R}_{k}-\bar{V}_{k}^{-} \delta t_{k}\right)\right. \\
& +B_{T, k} B_{k-1, k}^{-1}\left(\delta \bar{R}_{k-1}-\bar{V}_{k-1}^{+} \delta t_{k}\right)+B_{T, k}\left(\bar{a}_{k}^{-}-\bar{a}_{k}^{+}\right) \delta t_{k} \\
& +E_{T, k}\left(\delta m_{k}+\dot{m}_{g_{k}}^{+} \delta t_{k}\right)-A_{T, k+1}\left(\delta \bar{R}_{k+1}-\bar{V}_{k+1}^{-} \delta t_{k+1}\right) \\
& \left.+B_{T, k+1} \bar{a}_{k+1}^{-} \delta t_{k+1}\right] .
\end{aligned}
$$

It should be noted that the term $\delta m_{k}$ is still present in this expression; this term is a function of the control variables at previous maneuver patch points and will be dealt with at the end of this derivation. Combining like terms reduces Equation (39) to

$$
\begin{aligned}
\left(\bar{a}_{T}^{-}-\bar{a}_{T}^{+}\right) \delta t_{T} & =-\left(D_{T, k} B_{k-1, k}^{-1}-\tilde{S} B_{T, k} B_{k-1, k}^{-1}\right)\left(\delta \bar{R}_{k-1}-\bar{V}_{k-1}^{+} \delta t_{k}\right) \\
& -\left[\left(C_{T, k}+D_{T, k} D_{k, k-1} B_{k, k-1}^{-1}\right)-\tilde{S}\left(A_{T, k}+B_{T, k} D_{k, k-1} B_{k, k-1}^{-1}\right)\right]\left(\delta \bar{R}_{k}-\bar{V}_{k}^{-} \delta t_{k}\right) \\
& -\left(D_{T, k}-\tilde{S} B_{T, k}\right)\left(\bar{a}_{k}^{-}-\bar{a}_{k}^{+}\right) \delta t_{k}-\left(H_{T, k}-\tilde{S} E_{T, k}\right)\left(\delta m_{k}+\dot{m}_{g_{k}}^{+} \delta t_{k}\right) \\
& +\left(C_{T, k+1}-\tilde{S} A_{T, k+1}\right)\left(\delta \bar{R}_{k+1}-\bar{V}_{k+1}^{-} \delta t_{k+1}\right) \\
& -\left(D_{T, k+1}-\tilde{S} B_{T, k+1}\right) \bar{a}_{k+1}^{-} \delta t_{k+1} .
\end{aligned}
$$

Finally, let $\Delta \bar{a}_{T}=\bar{a}_{T}^{-}-\bar{a}_{T}^{+}$. This term can be written in terms of its magnitude and direction;

$$
\Delta \bar{a}_{T}=\left\|\Delta \bar{a}_{T}\right\| \frac{\Delta \bar{a}_{T}}{\left\|\Delta \bar{a}_{T}\right\|} .
$$

At point $T, \Delta \bar{a}_{T}$ is equal to the applied thrust. Thus, the unit vector $\frac{\Delta \bar{a}_{T}}{\left\|\Delta \bar{a}_{T}\right\|}$ is equal to the unit thrust 
direction $\hat{u}_{k}=\frac{\bar{u}_{k}^{+}}{\left\|\bar{u}_{k}^{+}\right\|}$. Equation (40) can therefore be written as

$$
\begin{aligned}
\left\|\Delta \bar{a}_{T}\right\| \hat{u}_{k} \delta t_{T} & =-\left(D_{T, k} B_{k-1, k}^{-1}-\tilde{S} B_{T, k} B_{k-1, k}^{-1}\right)\left(\delta \bar{R}_{k-1}-\bar{V}_{k-1}^{+} \delta t_{k}\right) \\
& -\left[\left(C_{T, k}+D_{T, k} D_{k, k-1} B_{k, k-1}^{-1}\right)-\tilde{S}\left(A_{T, k}+B_{T, k} D_{k, k-1} B_{k, k-1}^{-1}\right)\right]\left(\delta \bar{R}_{k}-\bar{V}_{k}^{-} \delta t_{k}\right) \\
& -\left(D_{T, k}-\tilde{S} B_{T, k}\right)\left(\bar{a}_{k}^{-}-\bar{a}_{k}^{+}\right) \delta t_{k}-\left(H_{T, k}-\tilde{S} E_{T, k}\right)\left(\delta m_{k}+\dot{m}_{g_{k}}^{+} \delta t_{k}\right) \\
& +\left(C_{T, k+1}-\tilde{S} A_{T, k+1}\right)\left(\delta \bar{R}_{k+1}-\bar{V}_{k+1}^{-} \delta t_{k+1}\right) \\
& -\left(D_{T, k+1}-\tilde{S} B_{T, k+1}\right) \bar{a}_{k+1}^{-} \delta t_{k+1} .
\end{aligned}
$$

Dividing both sides of Equation (42) by $\left\|\Delta \bar{a}_{T}\right\|$,

$$
\begin{aligned}
\hat{u}_{k} \delta t_{T} & =\left\|\Delta \bar{a}_{T}\right\|^{-1}\left[-\left(D_{T, k} B_{k-1, k}^{-1}-\tilde{S} B_{T, k} B_{k-1, k}^{-1}\right)\left(\delta \bar{R}_{k-1}-\bar{V}_{k-1}^{+} \delta t_{k}\right)\right. \\
& -\left[\left(C_{T, k}+D_{T, k} D_{k, k-1} B_{k, k-1}^{-1}\right)-\tilde{S}\left(A_{T, k}+B_{T, k} D_{k, k-1} B_{k, k-1}^{-1}\right)\right]\left(\delta \bar{R}_{k}-\bar{V}_{k}^{-} \delta t_{k}\right) \\
& -\left(D_{T, k}-\tilde{S} B_{T, k}\right)\left(\bar{a}_{k}^{-}-\bar{a}_{k}^{+}\right) \delta t_{k}-\left(H_{T, k}-\tilde{S} E_{T, k}\right)\left(\delta m_{k}+\dot{m}_{g_{k}}^{+} \delta t_{k}\right) \\
& +\left(C_{T, k+1}-\tilde{S} A_{T, k+1}\right)\left(\delta \bar{R}_{k+1}-\bar{V}_{k+1}^{-} \delta t_{k+1}\right) \\
& \left.-\left(D_{T, k+1}-\tilde{S} B_{T, k+1}\right) \bar{a}_{k+1}^{-} \delta t_{k+1}\right] .
\end{aligned}
$$

Because $\hat{u}_{k}$ is a unit vector, both sides of Equation (43) can be pre-multiplied by $\hat{u}_{k}^{T}$ to give

$$
\begin{aligned}
\delta t_{T} & =\frac{\hat{u}_{k}^{T}}{\left\|\Delta \bar{a}_{T}\right\|}\left[-\left(D_{T, k} B_{k-1, k}^{-1}-\tilde{S} B_{T, k} B_{k-1, k}^{-1}\right)\left(\delta \bar{R}_{k-1}-\bar{V}_{k-1}^{+} \delta t_{k}\right)\right. \\
& -\left[\left(C_{T, k}+D_{T, k} D_{k, k-1} B_{k, k-1}^{-1}\right)-\tilde{S}\left(A_{T, k}+B_{T, k} D_{k, k-1} B_{k, k-1}^{-1}\right)\right]\left(\delta \bar{R}_{k}-\bar{V}_{k}^{-} \delta t_{k}\right) \\
& -\left(D_{T, k}-\tilde{S} B_{T, k}\right)\left(\bar{a}_{k}^{-}-\bar{a}_{k}^{+}\right) \delta t_{k}-\left(H_{T, k}-\tilde{S} E_{T, k}\right)\left(\delta m_{k}+\dot{m}_{g_{k}}^{+} \delta t_{k}\right) \\
& +\left(C_{T, k+1}-\tilde{S} A_{T, k+1}\right)\left(\delta \bar{R}_{k+1}-\bar{V}_{k+1}^{-} \delta t_{k+1}\right) \\
& \left.-\left(D_{T, k+1}-\tilde{S} B_{T, k+1}\right) \bar{a}_{k+1}^{-} \delta t_{k+1}\right] .
\end{aligned}
$$

Using Equation (44) and recalling that $\Delta t_{b u r n_{n}}=t_{T}-t_{k}$, the partial derivatives of $\Delta t_{b u r n_{n}}$ with respect to the control variables are

$$
\begin{aligned}
\frac{\partial \Delta t_{b u r n_{n}}}{\partial \bar{R}_{k-1}} & =-\frac{\hat{u}_{k}^{T}}{\left\|\Delta \bar{a}_{T}\right\|}\left(D_{T, k} B_{k-1, k}^{-1}-\tilde{S} B_{T, k} B_{k-1, k}^{-1}\right) \\
\frac{\partial \Delta t_{b u r n_{n}}}{\partial t_{k-1}} & =\frac{\hat{u}_{k}^{T}}{\left\|\Delta \bar{a}_{T}\right\|}\left(D_{T, k} B_{k-1, k}^{-1}-\tilde{S} B_{T, k} B_{k-1, k}^{-1}\right) \bar{V}_{k-1}^{+} \\
\frac{\partial \Delta t_{b u r n_{n}}}{\partial \bar{R}_{k}} & =-\frac{\hat{u}_{k}^{T}}{\left\|\Delta \bar{a}_{T}\right\|}\left[\left(C_{T, k}+D_{T, k} D_{k, k-1} B_{k, k-1}^{-1}\right)-\tilde{S}\left(A_{T, k}+B_{T, k} D_{k, k-1} B_{k, k-1}^{-1}\right)\right] \\
\frac{\partial \Delta t_{b u r n_{n}}}{\partial t_{k}} & =\frac{\hat{u}_{k}^{T}}{\left\|\Delta \bar{a}_{T}\right\|}\left(\left[\left(C_{T, k}+D_{T, k} D_{k, k-1} B_{k, k-1}^{-1}\right)-\tilde{S}\left(A_{T, k}+B_{T, k} D_{k, k-1} B_{k, k-1}^{-1}\right)\right] \bar{V}_{k}^{-}\right. \\
& \left.-\left(D_{T, k}-\tilde{S} B_{T, k}\right)\left(\bar{a}_{k}^{-}-\bar{a}_{k}^{+}\right)-\left(H_{T, k}-\tilde{S} E_{T, k}\right) \dot{m}_{g}\right)-1, \\
\frac{\partial \Delta t_{b u r n_{n}}}{\partial \bar{R}_{k+1}} & =\frac{\hat{u}_{k}^{T}}{\left\|\Delta \bar{a}_{T}\right\|}\left(C_{T, k+1}-\tilde{S} A_{T, k+1}\right), \\
\frac{\partial \Delta t_{b u r n_{n}}}{\partial t_{k+1}} & =-\frac{\hat{u}_{k}^{T}}{\left\|\Delta \bar{a}_{T}\right\|}\left[\left(C_{T, k+1}-\tilde{S} A_{T, k+1}\right) \bar{V}_{k+1}^{-}+\left(D_{T, k+1}-\tilde{S} B_{T, k+1}\right) \bar{a}_{k+1}^{-}\right]
\end{aligned}
$$

The term containing $\delta m_{k}$ can be expressed as a function of the change in the initial mass, $\delta m_{0}$, and the burn durations of all maneuvers prior to patch point $k$,

$$
\delta m_{k}=\delta m_{0}-\sum_{j=1}^{n-1} \dot{m}_{g} \Delta t_{b u r n_{j}}
$$


where $j$ denotes the maneuver number and $n$ is the maneuver number at patch point $k$. Because the initial mass is fixed, this equation reduces to

$$
\delta m_{k}=-\sum_{j=1}^{n-1} \dot{m}_{g} \Delta t_{b u r n_{j}}
$$

Since $\dot{m}_{g}$ is a fixed, constant value, $\delta m_{k}$ is a function only of the previous burn durations. Thus, $\Delta t_{b u r n_{n}}$ has a dependence on the positions and times associated with any previous maneuvers that have occurred. Also, $\Delta V_{k}$ has a direct dependence on the mass at the beginning of the burn (and therefore on the previous burn durations), so that must be taken into account as well. Using the chain rule, the final form of the partial derivative of the constraint $\alpha$ (the sum of all the burn $\Delta V \mathrm{~s}$ ) with respect to any control variable $\beta_{k}$ in the set of control variables associated with patch point $k$ is

$$
\frac{\partial \alpha}{\partial \beta_{k}}=\frac{\partial \Delta V_{n}}{\partial \Delta t_{b u r n_{n}}} \frac{\partial \Delta t_{\text {burn }}}{\partial \beta_{k}}+\sum_{j=n+1}^{N} \frac{\partial \Delta V_{j}}{\partial \Delta t_{b u r n_{n}}} \frac{\partial \Delta t_{b u r n_{n}}}{\partial \beta_{k}}
$$

where

$$
\frac{\partial \Delta V_{j}}{\partial \Delta t_{b u r n_{n}}}=\frac{\partial \Delta V_{j}}{\partial \Delta t_{b u r n_{n}}}+\frac{\partial \Delta V_{j}}{\partial \Delta t_{b u r n_{j}}} \frac{\partial \Delta t_{b_{\text {burn }}}}{\partial \Delta t_{b u r n_{n}}} .
$$

These partial derivatives are then placed into the $M$ matrix in accordance with the existing maneuver constraint formulation.

\section{Simulation and Results}

The two level targeting algorithm is applied in the following section in both the impulsive and finite burn configurations. This is done in order to demonstrate its performance under both configurations and to gain insight as to applications of each. Then, as a final metric, an optimal trajectory is generated to use for performance comparisons.

For each case, the same initial conditions of the lunar orbit will be used as follows:

- Epoch: 4-Apr-2024 15:30:00 TDT

- Initial mass: $20339.9 \mathrm{~kg}$ (total fuel $=8063.65 \mathrm{~kg}$ )

- Main Engine Thrust: 33,361.6621 N

- Main Engine Isp: $326 \mathrm{sec}$

- Auxiliary Engine Thrust: 4,448.0 N

- Auxiliary Engine Isp: $309 \mathrm{sec}$

- State (J2000 Moon-centered inertial frame):

- X: - $1236.7970783385588 \mathrm{~km}$

- Y: $1268.1142350088496 \mathrm{~km}$

- Z: $468.38317094160635 \mathrm{~km}$

- Vx: $0.0329108058365355 \mathrm{~km} / \mathrm{sec}$

- Vy: $0.589269803607714 \mathrm{~km} / \mathrm{sec}$

- Vz - $1.528058717568413 \mathrm{~km} / \mathrm{sec}$

Likewise, the same terminal target conditions will be used for each case as shown below:

- Geodetic Altitude (km) 121.92

- Longitude (deg) 175.6365

- Geocentric Azimuth (deg) 49.3291

- Geocentric Flight Path Angle (deg) -5.86 


\section{A. Impulsive Algorithm Example}

The first case is representative of a nominal Earth return during which the maneuvers are performed by the CM main engine. For this case, the impulsive targeter is executed and the initial guess file consists of 12 "patch points" or states taken from an optimized trajectory. The first patch point corresponds to a state ad epoch 20 minutes prior to the optimized TEI -1 TIG. The interior patch points correspond to the states and epochs at each of maneuver locations (TEI-1, 2, 3 and TCM 1,2,3) and some additional waypoints along the trajectory. The final patch point in the initial guess is the state and epoch at the desired entry interface (EI).

For this case the impulsive targeter is executed in order to find a feasible trajectory that satisfies the specified terminal constraints, while minimizing the $\Delta V$ sum of the individual maneuvers. The targeter converges on a feasible solution after 15 iterations and 2.34 minutes of computation time (Figure 2). The total $\Delta V$ of this solution is $1.3484 \mathrm{~km} / \mathrm{s}$. The $\Delta V$ for each maneuver is given in Table 1 .

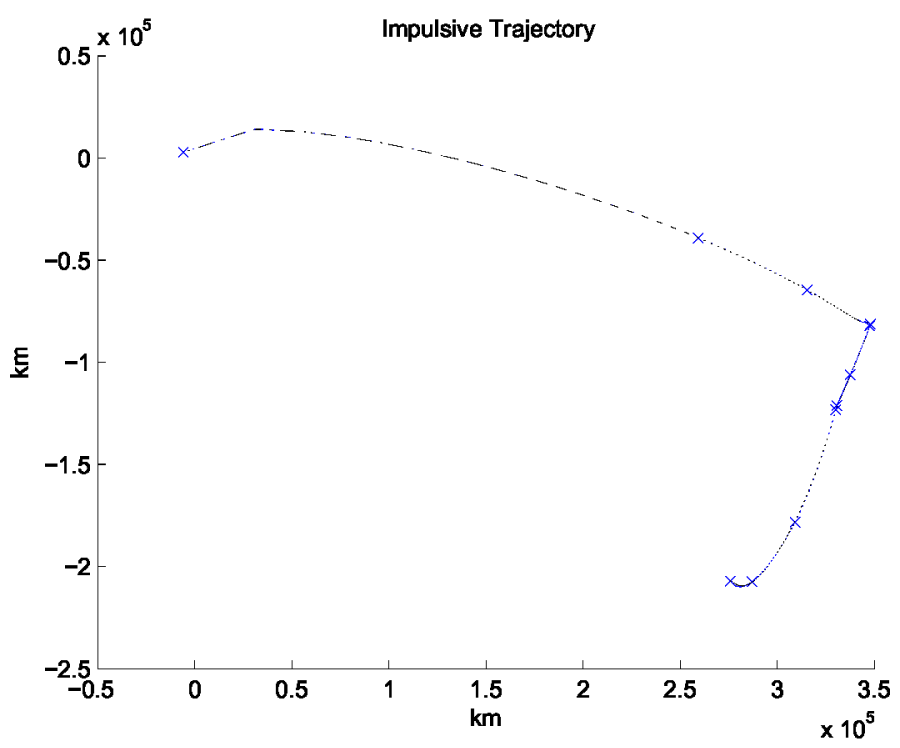

Figure 2. Initial Guess and Impulsive Solution

Table 1. Maneuver Data

\begin{tabular}{|c|c|}
\hline \hline Maneuver & $\Delta V(\mathrm{~km} / \mathrm{s})$ \\
\hline TEI-1 & 0.6753 \\
TEI-2 & 0.2617 \\
TEI-3 & 0.4114 \\
\hline
\end{tabular}

\section{B. Finite Burn Algorithm Example}

Assuming the use of the main engines for all three maneuvers, the finite burn algorithm is able to converge on a final solution in 24 iterations, with a total $\Delta V$ of $1.3353 \mathrm{~km} / \mathrm{s}$. The maneuver sum constraint is active for this case, with a maximum allowable $\Delta V$ of $1.40 \mathrm{~km} / \mathrm{s}$. The full solution is shown in Figure 3. Figures 4 , 5 , and 6 show closeup views of TEI-1, TEI-2, and TEI-3, respectively. The solid red sections in each of these closeups indicate the thruster burn segments of each arc. The burn duration, propellant mass consumption, and $\Delta V$ for each maneuver are given in Table 2 . The total computation time required to achieve a solution is 6.57 minutes. 


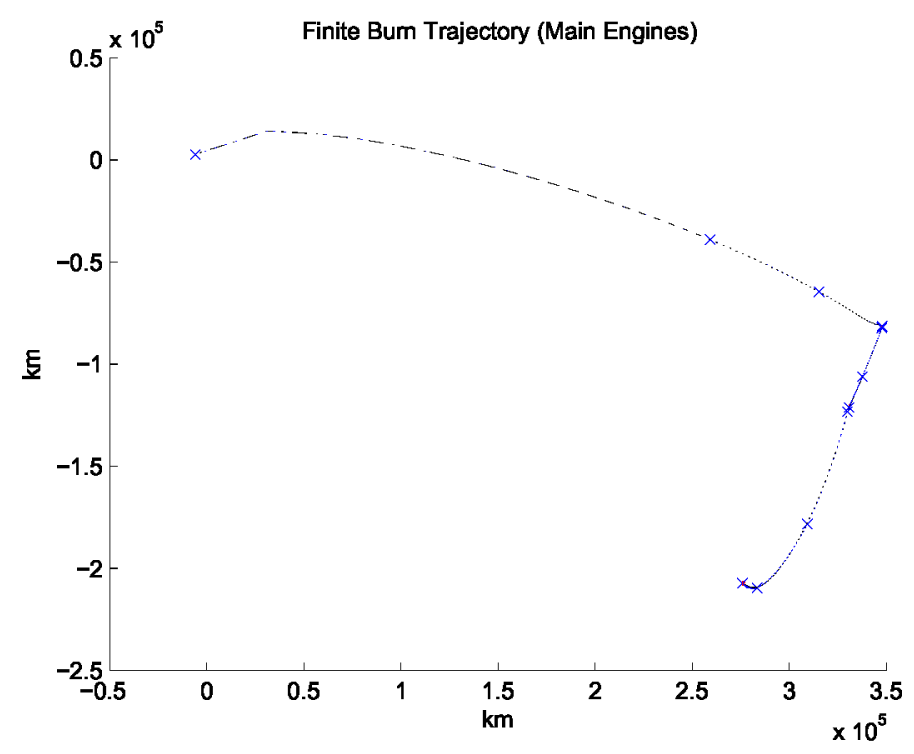

Figure 3. Initial Guess and Finite Burn Solution with Main Engines, Full Trajectory

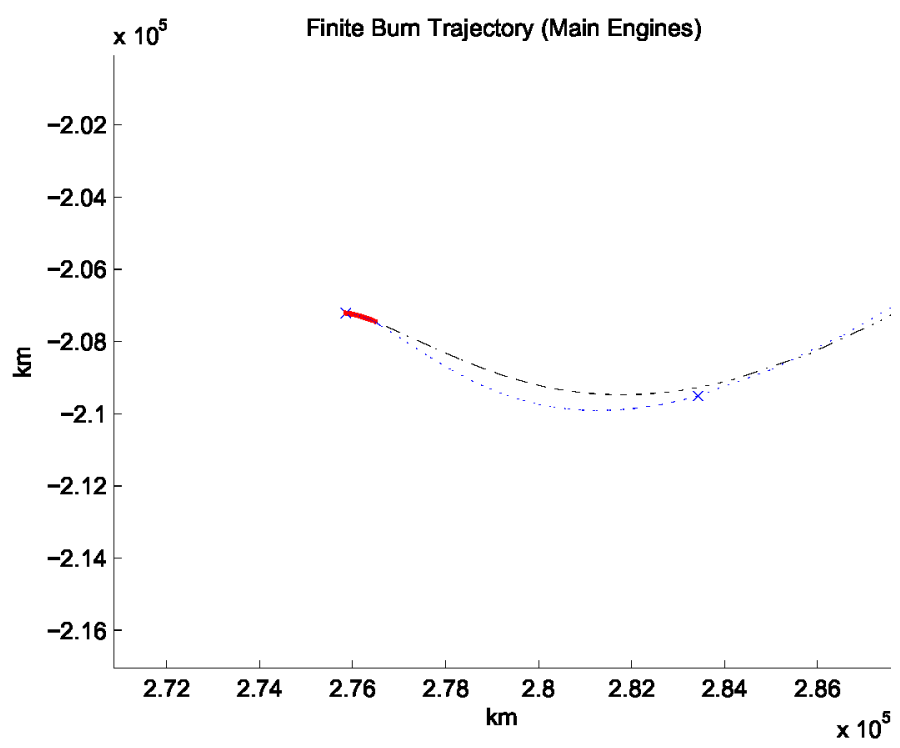

Figure 4. Initial Guess and Finite Burn Solution with Main Engines, TEI-1 Closeup

Table 2. Burn Data Using Main Engines

\begin{tabular}{|c|c|c|c|}
\hline \hline Maneuver & Duration (s) & Prop. Mass Consumed (kg) & $\Delta V(\mathrm{~km} / \mathrm{s})$ \\
\hline TEI-1 & 377.6462 & 3939.530 & 0.6546 \\
TEI-2 & 131.2084 & 1368.740 & 0.2650 \\
TEI-3 & 184.1434 & 1920.947 & 0.4157 \\
\hline
\end{tabular}




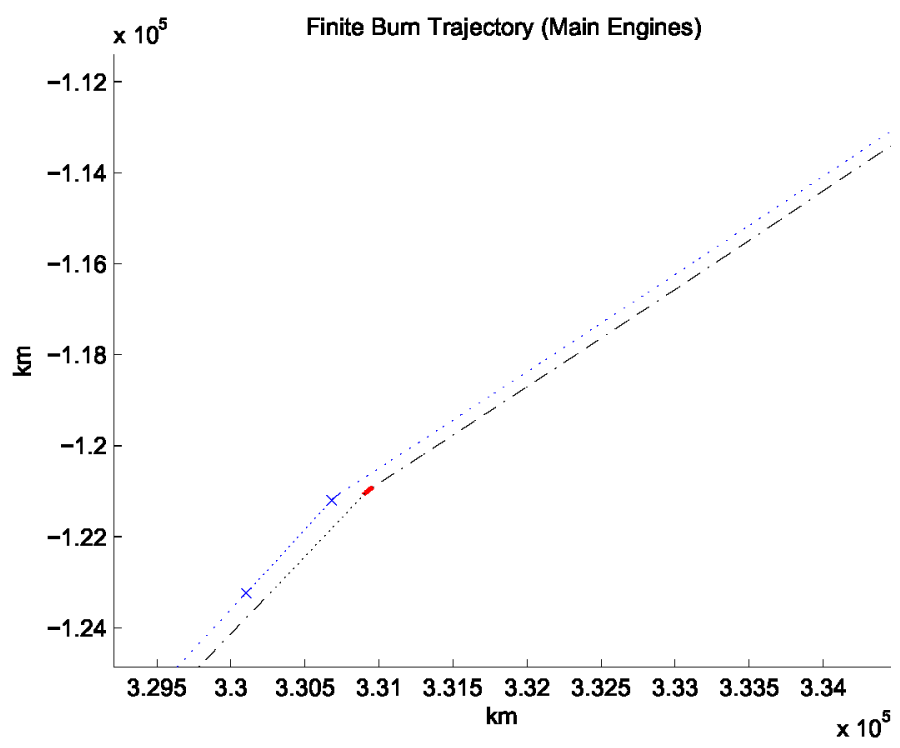

Figure 5. Initial Guess and Finite Burn Solution with Main Engines, TEI-2 Closeup

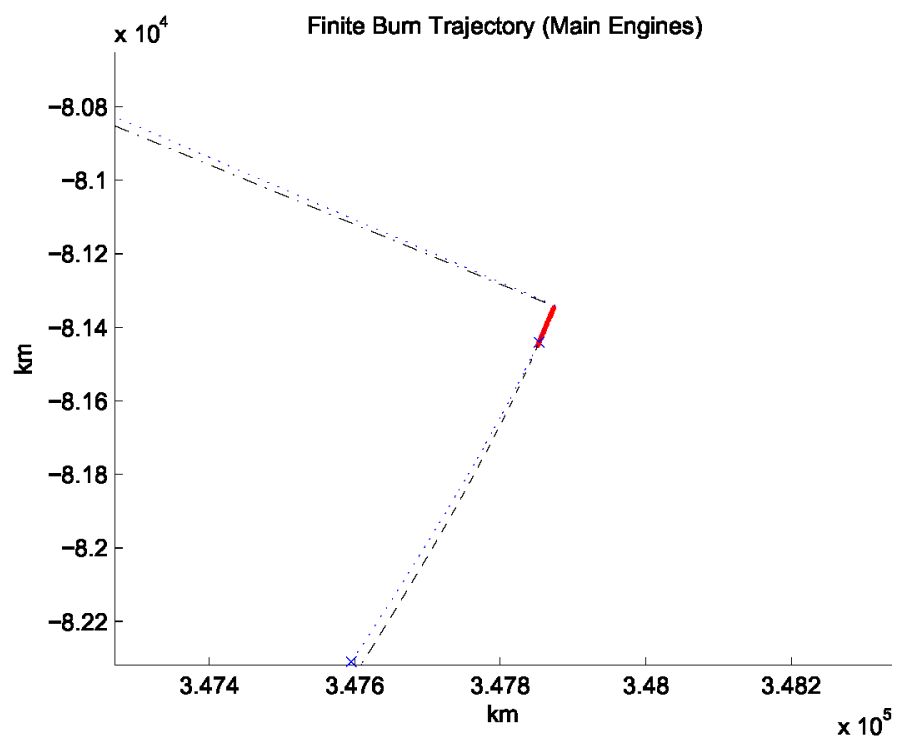

Figure 6. Initial Guess and Finite Burn Solution with Main Engines, TEI-3 Closeup 


\section{Finite Burn Algorithm with Auxiliary Engines}

For this case, the maneuver sum constraint is relaxed to a maximum $\Delta V$ of $1.50 \mathrm{~km} / \mathrm{s}$. Using only the auxiliary engines, the algorithm converges in 18 iterations, with a total $\Delta V$ of $1.500 \mathrm{~km} / \mathrm{s}$. As in the previous section, the solid red portions of the final trajectory closeups indicate the segments of the trajectory in which the engines are thrusting. Figure 7 shows the impulsive initial guess and the final finite burn solution for the full return trajectory. Figures 8 through 10 show the closeup views of TEI-1, TEI-2, and TEI-3. Burn data for each maneuver is listed in Table 3. The solution is identified after 7.67 minutes of computation time.

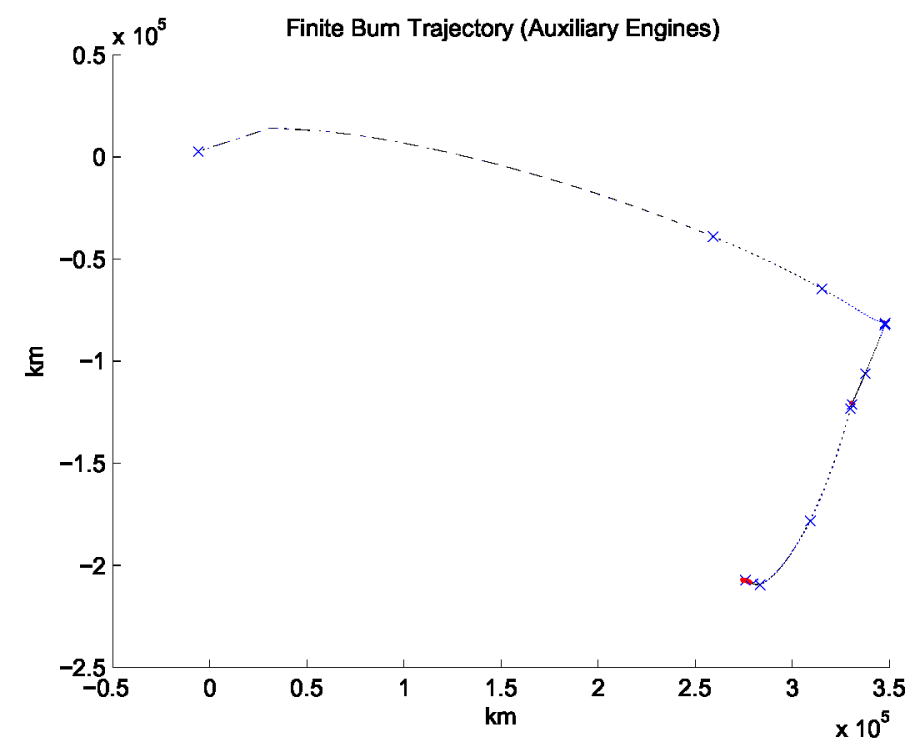

Figure 7. Initial Guess and Finite Burn Solution with Auxiliary Engines, Full Trajectory

Table 3. Burn Data Using Auxiliary Engines

\begin{tabular}{|c|c|c|c|}
\hline \hline Maneuver & Duration (s) & Prop. Mass Consumed $(\mathrm{kg})$ & $\Delta V(\mathrm{~km} / \mathrm{s})$ \\
\hline TEI-1 & 3220.3465 & 4725.536 & 0.8039 \\
TEI-2 & 934.8663 & 1371.823 & 0.2796 \\
TEI-3 & 1242.5484 & 1823.316 & 0.4165 \\
\hline
\end{tabular}

\section{Optimized Finite Burn Trajectory}

As a final step in this analysis, the previous trajectory generated from the finite burn targeter with auxiliary thrusters was optimized using Copernicus. Again, the same initial conditions and initial guess file were used to initiate both runs. The results of this optimized run are available in Table 4 . The total $\Delta V$ for the optimal run is $1.2413 \mathrm{~km} / \mathrm{s}$. This is an improvement of approximately $0.25 \mathrm{~km} / \mathrm{s}$ of $\Delta V$ over the finite burn targeting solution.

Table 4. Optimal Burn Data Using Auxiliary Engines

\begin{tabular}{|c|c|c|c|}
\hline \hline Maneuver & Duration (s) & Prop. Mass Consumed $(\mathrm{kg})$ & $\Delta V(\mathrm{~km} / \mathrm{s})$ \\
\hline TEI-1 & 3327.008 & 3501.37 & 0.6040 \\
TEI-2 & 873.040 & 1504.85 & 0.2993 \\
TEI-3 & 1312.285 & 1828.22 & 0.4059 \\
\hline
\end{tabular}




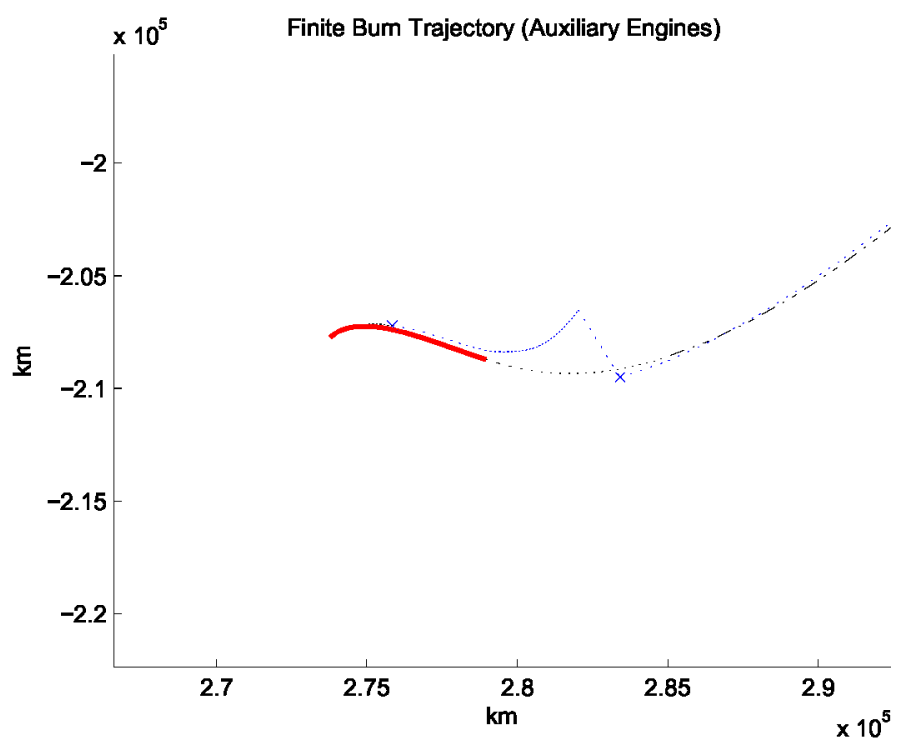

Figure 8. Initial Guess and Finite Burn Solution with Auxiliary Engines, TEI-1 Closeup

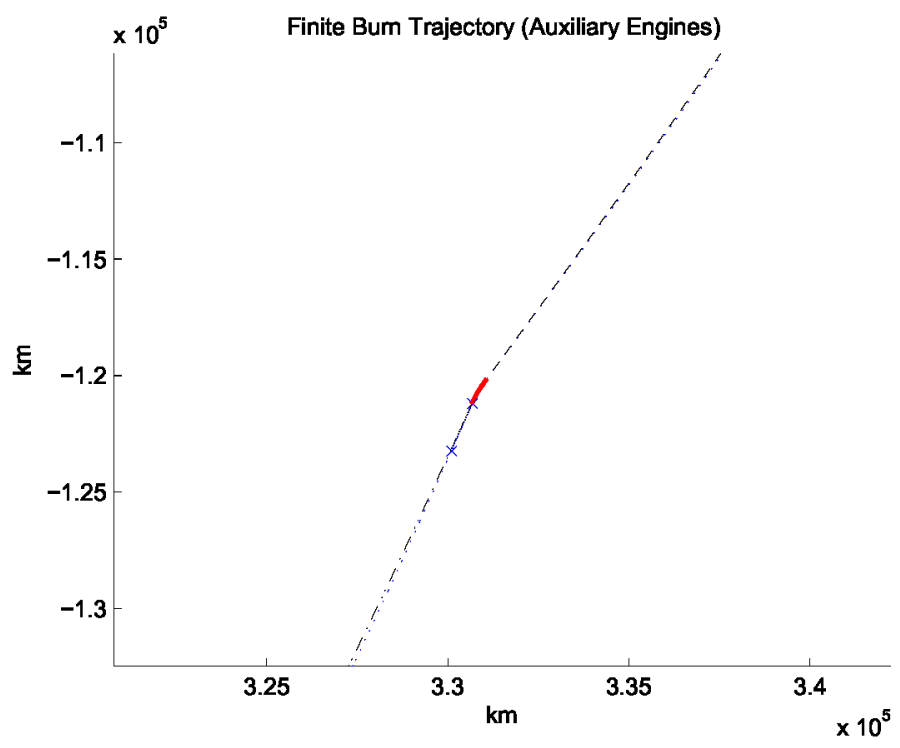

Figure 9. Initial Guess and Finite Burn Solution with Auxiliary Engines, TEI-2 Closeup 


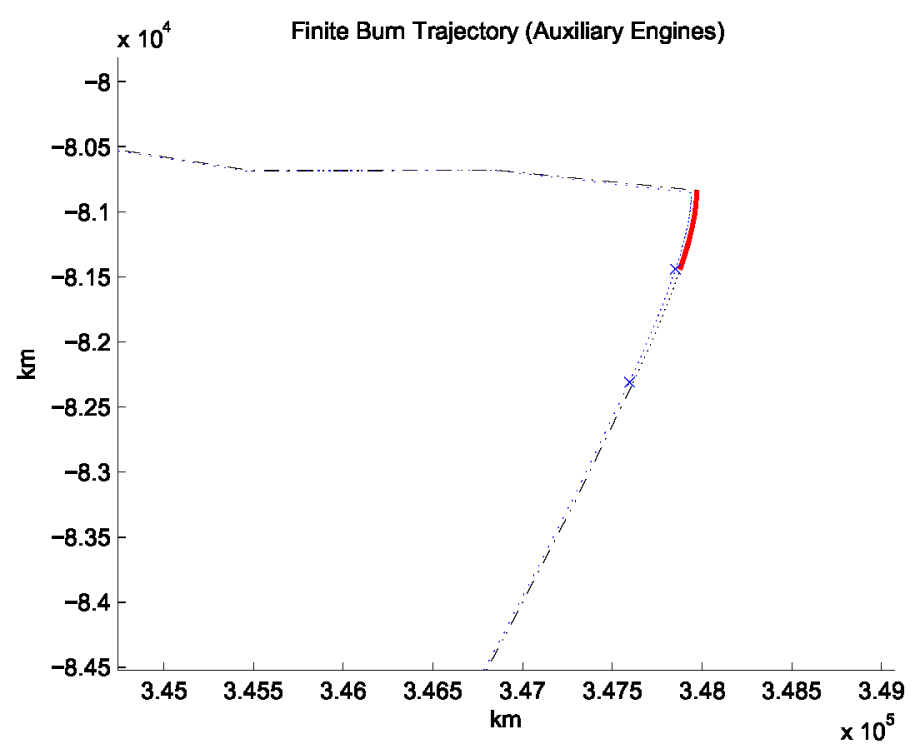

Figure 10. Initial Guess and Finite Burn Solution with Auxiliary Engines, TEI-3 Closeup

\section{Conclusions}

This paper presents a two-level targeting algorithm for finite burn maneuvers. The algorithm is a modification of the impulsive targeting scheme described in Reference 2. Formulations for the finite burn versions of both the Level I and Level II process are discussed, and a total mission cost constraint, originally developed for the impulsive algorithm, is modified and adapted to the finite burn problem. The algorithm is tested for the trans-Earth injection phase of the Orion mission. Results are compared for three different cases: using the impulsive algorithm, using the finite burn algorithm with main engines for all three maneuvers, and using the finite burn algorithm with only the auxiliary engines for the final two maneuvers. Results show that the finite burn algorithm is able to converge on a feasible solution even in the case of a main engine failure following TEI-1. Due to the additional complexity of the finite burn equations, the algorithm requires more computational time than the impulsive targeter, but it still shows significant improvement over an optimizer in terms of the computation cost.

\section{References}

\footnotetext{
${ }^{1}$ Marchand, B., Howell, K., and Wilson, R., "Improved Corrections Process for Constrained Trajectory Design in the n-Body Problem," Journal of Spacecraft and Rockets, Vol. 44, No. 4, 2007, pp. 884-897.

${ }^{2}$ Weeks, M. W., Marchand, B. G., Smith, C. W., and Scarritt, S., "Design of the Onboard Autonomous Targeting Algorithm for the Trans-Earth Phase of Orion," AIAA Guidance, Navigation, and Control Conference and Exhibit, Honolulu, HI, Aug. 2008, AIAA-2008-7262.

${ }^{3}$ Smith, C. W., An Onboard Targeting Algorithm with Earth-Return Applications, Master's thesis, University of Texas at Austin, August 2008.
} 